\section{A case of invasive pulmonary aspergillosis during treatment for acute exacerbation of interstitial lung disease}

\author{
Motoi Ugajin, ${ }^{1}$ Hisanori Kani ${ }^{2}$ \\ Departments of ${ }^{1}$ Respiratory Medicine \\ and ${ }^{2}$ Thoracic Surgery, Nagoya \\ Tokushukai General Hospital, Japan
}

\begin{abstract}
Prolonged immunosuppressive therapy is a risk factor for invasive pulmonary aspergillosis. We report a case of a 79-yearold man who underwent immunosuppressive therapy with methylprednisolone and cyclosporine for an acute exacerbation of interstitial lung disease. Ten days after initiation of immunosuppressive therapy, the patient reported night sweats and purulent sputum, and chest computed tomography scan revealed consolidation. He was diagnosed with invasive pulmonary aspergillosis, and required vasopressor support with oxygen therapy. After the administration of voriconazole and the modulation of immunosuppressive therapy, his condition improved. Short-term immunosuppressive therapy can also induce invasive pulmonary aspergillosis.
\end{abstract}

\section{Introduction}

Invasive pulmonary aspergillosis (IPA) is an opportunistic infection. The mortality rate of IPA is reported to exceed $50 \%$ due to the difficulty of its diagnosis and the rapid progression of its clinical course. ${ }^{1}$ Prolonged immunosuppressive therapy is recognized as a significant risk factor of IPA. ${ }^{2}$ However, the occurrence of IPA in patients after short-term immunosuppressive therapy for lung diseases is rare. Herein, we present a case of IPA that was diagnosed 10 days after the initiation of immunosuppressive therapy for an acute exacerbation of interstitial lung disease.

\section{Case Report}

A 79-year-old man arrived at our emergency room by ambulance complaining of dyspnea and non-productive cough. He was seen at our hospital every 2 months for interstitial lung disease and his lung function test performed 8 months previously showed that his forced vital capacity was
$2.26 \mathrm{~L}(67.0 \%$ of the predicted value). His other medical history was angina pectoris treated with percutaneous coronary intervention 2 years previously. In the emergency room, his respiratory rate was 30 breaths/min and his oxygen saturation was $92 \%$ using a face mask with oxygen flow of $6 \mathrm{~L} / \mathrm{min}$. Fine crackles were heard in both lungs on chest auscultation and chest computed tomography (CT) revealed groundglass opacities and reticular infiltrates in both lung fields (Figure 1A). He was diagnosed with an acute exacerbation of interstitial lung disease and was administered intravenous methylprednisolone $500 \mathrm{mg}$ once daily with levofloxacin. His general condition improved and his chest CT on day 4 of admission showed improvement of ground-glass opacities and reticular infiltrates in both lung fields (Figure 1B). The dose of intravenous methylprednisolone was reduced to $40 \mathrm{mg}$ once daily and the patient was started on oral cyclosporine 50 mg twice daily. On day 11 of admission, he reported night sweats and purulent sputum. At that time, his serum C-reactive protein level was $13.17 \mathrm{mg} / \mathrm{dL}$ (normal value $<0.3$ $\mathrm{mg} / \mathrm{dL}$ ) and chest CT showed a wedgeshaped consolidation in the right lower lobe (Figure 1C). The patient was initiated on intravenous micafungin $150 \mathrm{mg}$ once daily with piperacillin/tazobactam. However, his condition deteriorated and he was transferred to the intensive care unit on day 18 of admission for vasopressor support with oxygen therapy. His chest CT on day 18 of admission showed an obvious enlargement of the consolidation in the right lower lobe (Figure 1D). His serum $\beta$-D glucan level was elevated $(1,662 \mathrm{pg} / \mathrm{mL}$, normal value $<$ $20 \mathrm{pg} / \mathrm{mL}$ ) and serum aspergillus galactomannan antigen was positive (3.9, cut-off value of 0.5 ). He was clinically diagnosed with IPA and treated with intravenous voriconazole $300 \mathrm{mg}$ twice daily, followed by $200 \mathrm{mg}$ twice daily starting the following day. His cyclosporine was stopped and the intravenous methylprednisolone was reduced to $20 \mathrm{mg}$ once daily. After initiation of voriconazole, his condition gradually improved. His serum $\beta$-D glucan level and serum aspergillus galactomannan antigen index also decreased (Table 1). A bronchoscopy was performed on day 37 of admission and bronchial lavage was obtained from the right lower bronchus. The bronchial lavage revealed filamentous fungi (Figure 2) and Aspergillus fumigatus was cultivated. On day 40 of admission, the intravenous voriconazole was switched to oral administration $200 \mathrm{mg}$ twice daily. On day 51 of admission, he was discharged with marked shrinkage of the consolidation in his right lower lobe.
Correspondence: Motoi Ugajin, Department of Respiratory Medicine, Nagoya Tokushukai General Hospital, 2-52 Kozoji-cho-kita, Kasugai 487-0016, Japan.

Tel.: +81.568.518711 - Fax: +81.568.517115. E-mail: ugarin2001@yahoo.co.jp

Key words: Invasive pulmonary aspergillosis; Interstitial lung disease; Immunosuppressive therapy.

Contributions: MU diagnosed and treated the patient, and wrote the manuscript; HK diagnosed the patient, and revised the manuscript.

Conflict of interest: the authors declare no potential conflict of interest.

Funding: none.

Received for publication: 2 July 2018.

Revision received: 14 September 2018.

Accepted for publication: 9 October 2018.

This work is licensed under a Creative Commons Attribution-NonCommercial 4.0 International License (CC BY-NC 4.0).

CC Copyright M. Ugajin and H. Kani, 2018 Licensee PAGEPress, Italy

Infectious Disease Reports 2018; 10:7785 doi:10.4081/idr.2018.7785

\section{Discussion}

The present case showed that even short-term immunosuppressive therapy can cause IPA. This life-threatening form of aspergillus infection has occasionally been seen in immunocompromised patients with a hematologic disorder or post-organ transplantation. ${ }^{2}$ Similarly, immunosuppressive therapy for lung disease is a known risk factor of IPA. However, most previous reports indicate that IPA occurred in patients after a prolonged term of immunosuppressive therapy at least 3 weeks. ${ }^{3,4}$ In the present case, IPA occurred only 10 days after initiation of immunosuppressive therapy. Hence, we propose that a short term of immunosuppressive therapy can induce IPA.

Voriconazole has been established as the primary antifungal agent for treatment of IPA. ${ }^{5}$ In fact, the present case showed remarkable clinical improvement after administration of voriconazole. However, voriconazole interacts with a considerable number of drugs. ${ }^{6}$ Serum cyclosporine concentration has been reported to be elevated after initiation of voriconazole and a high trough concentration of cyclosporine is associated with nephrotoxicity. ${ }^{7,8}$ Although re-exacerbation of interstitial lung disease 
Table 1. Transition of serum $\beta$-D glucan level and aspergillus galactomannan antigen index. Normal values: $\beta$-D glucan $<20 \mathrm{pg} / \mathrm{mL}$, galactomannan antigen index $<0.5$.

\begin{tabular}{lccccccc} 
& Day 2 & Day 11 & Day 18 & Day 25 & Day 32 & Day 39 \\
Serum $\beta$-D glucan level $(\mathrm{pg} / \mathrm{mL})$ & 12.4 & 1662.0 & 2665.0 & 209.7 & 67.0 & 34.4 \\
Galactomannnan antigen index & - & 3.9 & - & 0.9 & - & 0.5 \\
\hline
\end{tabular}

A
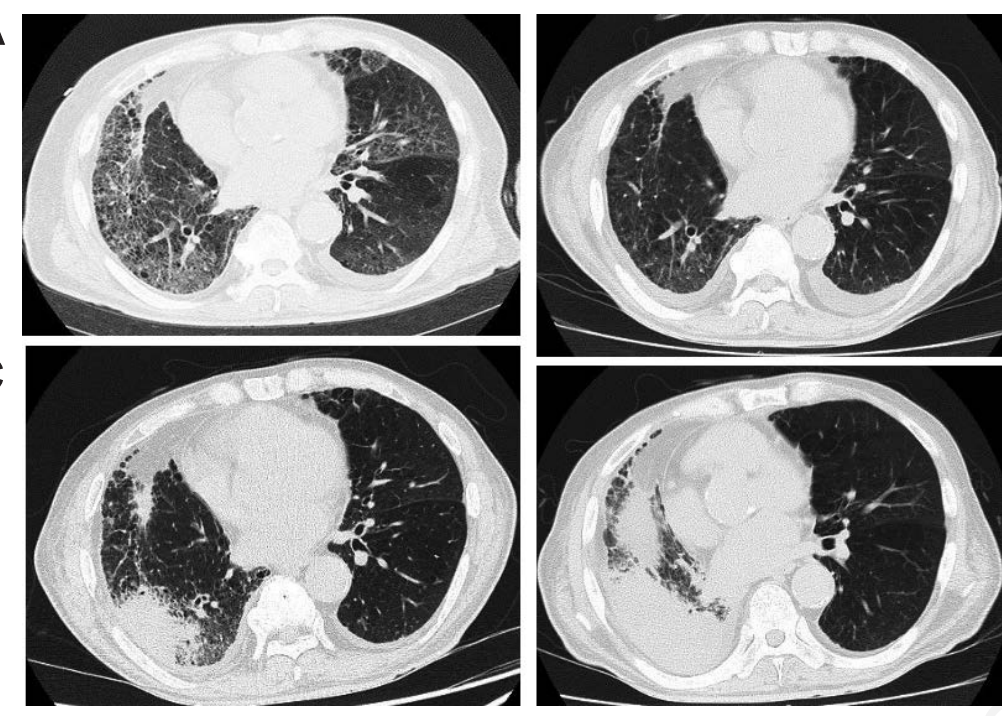

B

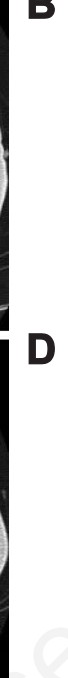

Figure 1. A) Ground-glass opacities and reticular infiltrates were observed in both lung fields on the day of admission. B) Ground-glass opacities and reticular infiltrates almost disappeared by day 4 of admission. C) A wedge-shaped consolidation was observed in the right lower lobe on day 11 of admission. D) Consolidation in the right lower lobe was enlarged on day 18 of admission.

was a concern, we decided to stop cyclosporine treatment and reduce the dose of methylprednisolone at initiation of voriconazole in order to avoid cyclosporine-induced nephrotoxicity and to restore host immune status. Kousha et al. assert the importance of decreasing the dosage of systemic corticosteroids and immunosuppressive agents in the treatment of patients with IPA. ${ }^{6}$ Owing to both the appropriate choice of antifungal agent and the modulation of immunosuppressive therapy, the present case had successful resolution of IPA.

It remains unclear why only some immunocompromised patients develop IPA while others do not. Host recognition of invading pathogen is an important first process against invasive infection. Recently, some genetic defects of the recognition process have been revealed and considered to be a factor of susceptibility to aspergillus infection. ${ }^{9}$ As an example, genetic Pentraxin 3 (PTX3) deficiency is known to be a risk factor of invasive aspergillosis. ${ }^{10}$ PTX3 is an inflammatory protein activating the toll-like receptor pathway by binding aspergillus conidia. ${ }^{11}$ Among recipients of hematopoietic stemcell transplantation, the incidence of invasive aspergillosis was significantly higher in case of genetic PTX3 deficient donors. ${ }^{12}$ A study in patients with chronic obstructive pulmonary disease showed that PTX3 rs1840680 single nucleotide polymorphism was associated with susceptibility to aspergillus infection and the polymorphism was detected in 31 out of 173 patients. ${ }^{13}$ Although we could not investigate genetic defects relating susceptibility to aspergillus infection in the present case, screening of genetic defects may be a potential useful tool to stratify the risk of IPA occurrence in initiating immunosuppressive therapy.

Neutrophils play a key role of the first line defense against aspergillus infection. ${ }^{14}$ To eliminate aspergillus germinating spores, neutrophils produce extracellular traps, release antimicrobial proteases by degranulation, and activate the toll-like receptor pathway using preliminarily stored PTX3. ${ }^{15-17}$ In the present case, patient neutrophil function was considered to be quite attenuated because of his high serum galactomannan index. Galactomannan, a component of the cell wall of Aspergillus spp., is released into blood stream during hyphal growth and is captured by neutrophils. It has been known that non-neutropenic patients with invasive aspergillosis have significant lower serum galactomannan indexes as compared to neutropenic

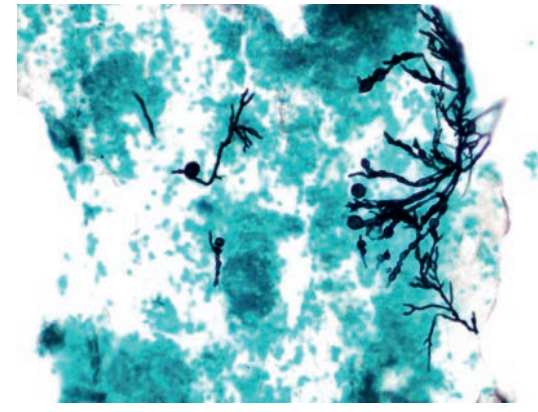

Figure 2. Black stained hyphae were observed in the right lower bronchial lavage (Grocott stain, 200× magnification).

patients. ${ }^{18}$ Zhou et al. reported that only 14 out of 37 IPA patients without neutropenia had positive serum galactomannan indexes at a cut-off value of 0.5 and the mean value of galactomannan index in the 37 IPA patients was $0.74 .{ }^{19}$ Despite a sufficient neutrophil count $(16,376 / \mathrm{uL})$, the present case had a high serum galactomannan index of 3.9 on day 11 of admission. The attenuation of neutrophil function was suggested in the present case and may be an important factor of IPA occurrence.

\section{Conclusions}

In conclusion, we presented a case of IPA associated with short-term immunosuppressive therapy for an acute exacerbation of interstitial lung disease. Adequate diagnosis, appropriate choice of antifungal agent, and careful assessment of host immune status are essential for the management of IPA.

\section{References}

1. Taccone FS, Van den Abeele AM, Bulpa $\mathrm{P}$, et al. Epidemiology of invasive aspergillosis in critically ill patients: clinical presentation, underlying conditions, and outcomes. Crit Care 2015;19:7.

2. Blot SI, Taccone FS, Van den Abeele $\mathrm{AM}$, et al. A clinical algorithm to diagnose invasive pulmonary aspergillosis in critically ill patients. Am J Respir 
Crit Care Med 2012;186:56-64.

3. Palmer LB, Greenberg HE, Schiff MJ. Corticosteroid treatment as a risk factor for invasive aspergillosis in patients with lung disease. Thorax 1991;46:1520.

4. Jonaitytė B, Kibarskytè R, Danila E, et al. Fatal pulmonary complication during induction therapy in a patient with ANCA-associated vasculitis. Acta Med Litu 2016;23:142-6.

5. See comment in PubMed Commons belowLimper AH, Knox KS, Sarosi GA, et al. An official American Thoracic Society statement: Treatment of fungal infections in adult pulmonary and critical care patients. Am J Respir Crit Care Med 2011;183:96-128.

6. Kousha M, Tadi R, Soubani AO. Pulmonary aspergillosis: a clinical review. Eur Respir Rev 2011;20:15674.

7. Mori T, Aisa Y, Kato J, et al. Drug interaction between voriconazole and calcineurin inhibitors in allogeneic hematopoietic stem cell transplant recipients. Bone Marrow Transplant 2009;44:371-4.

8. Jacobson PA, Schladt D, Israni A, et al. Genetic and clinical determinants of early, acute calcineurin inhibitor-related nephrotoxicity: results from a kidney transplant consortium. Transplantation 2012;93:624-31.

9. Campos CF, van de Veerdonk FL, Gonçalves SM, et al. Host Genetic Signatures of Susceptibility to Fungal Disease. Curr Top Microbiol Immunol 2018 Jul 25. [Epub ahead of print]

10. Maskarinec SA, Johnson MD, Perfect JR. Genetic Susceptibility to Fungal Infections: What is in the Genes? Curr Clin Microbiol Rep 2016;3:81-91.

11. Bozza S, Campo S, Arseni B, et al. PTX3 binds MD-2 and promotes TRIFdependent immune protection in aspergillosis. J Immunol 2014;193 2340-8.

12. Cunha C, Aversa F, Lacerda JF, et al. Genetic PTX3 deficiency and aspergillosis in stem-cell transplantation. N Engl J Med 2014; 370:421-32.

13. He Q, Li H, Rui Y, et al. Pentraxin 3 Gene Polymorphisms and Pulmonary Aspergillosis in Chronic Obstructive Pulmonary Disease Patients. Clin Infect Dis 2018;66:261-7.

14. Espinosa V, Rivera A. First Line of Defense: Innate Cell-Mediated Control of Pulmonary Aspergillosis. Front Microbiol 2016;7:272.

15. Bruns S, Kniemeyer O, Hasenberg M, et al. Production of extracellular traps against Aspergillus fumigatus in vitro and in infected lung tissue is dependent on invading neutrophils and influenced by hydrophobin RodA. PLoS Pathog 2010;6:e1000873.

16. Feldmesser M. Role of neutrophils in invasive aspergillosis. Infect Immun 2006; 74: 6514-6.

17. Jaillon S, Peri G, Delneste Y, et al. The humoral pattern recognition receptor PTX3 is stored in neutrophil granules and localizes in extracellular traps. J Exp Med 2007;204:793-804.

18. Cordonnier C, Botterel F, Ben Amor R, et al. Correlation between galactomannan antigen levels in serum and neutrophil counts in haematological patients with invasive aspergillosis. Clin Microbiol Infect 2009;15:81-6.

19. Zhou W, Li H, Zhang Y, et al. Diagnostic Value of Galactomannan Antigen Test in Serum and Bronchoalveolar Lavage Fluid Samples from Patients with Nonneutropenic Invasive Pulmonary Aspergillosis. J Clin Microbiol 2017;55:2153-61. 